\section{A team-based approach in primary care can alleviate the burden created by the COVID-19 surgical backlog for people with hip and knee osteoarthritis}

Wang and colleagues provided a timely estimate of the sizeable surgical backlog in Ontario created by coronavirus disease 2019 (COVID-19) and the substantial investment of resources required to clear it. ${ }^{1}$ Osteoarthritis $(\mathrm{OA})$ is associated with $\$ 1.4$ billion annually in direct health care costs. ${ }^{2}$ With more than 137000 hip and knee total joint replacements (TJRs) performed each year, ${ }^{2}$ patients waiting for TJR make up a sizable component of the COVID-19 surgical backlog. How best to optimize the health and quality of life for the thousands of individuals left in limbo while waiting for a rescheduled surgery? We propose that primary care clinicians, including physicians and physiotherapists, can work together to reduce pain and improve the quality of life for those with OA waiting for TJR.

International best practice guidelines for hip and knee OA strongly recommend patient education, exercise therapy and weight control (if necessary) as essential treatments. ${ }^{3,4}$ Even individuals with severe OA have the potential to improve their quality of life with comprehensive nonsurgical management. ${ }^{5}$ In a landmark study (MEDIC), ${ }^{5}$ patients with severe knee $O A$ who were eligible for TJR were randomized to either TJR followed by 12 weeks of nonsurgical treatment, or 12 weeks of nonsurgical treatment alone. Nonsurgical treatment was delivered by physiotherapists and dieticians, and consisted of exercise, education, dietary advice, insoles and pain medication. While those who had TJR experienced greater improvements in pain and function, patients who received only nonsurgical treatment also had clinically significant improvements at 12 months and $75 \%$ had postponed their TJR at least 1 year. ${ }^{5}$ Unfortunately, however, only half of Canadians with OA receive comprehensive nonsurgical management. ${ }^{6}$

Physiotherapists, as experts in exercise prescription and chronic disease management, are key team members in OA management. Physiotherapists can deliver comprehensive evidence-based programs that are available across Canada and have been proven to reduce pain, and improve function and quality of life, such as Good Life with Osteoarthritis Denmark (GLA:D). ${ }^{7,8}$ Despite strong evidence, physiotherapy for knee and hip OA is rarely publicly funded, and Canadians must often use extended health insurance or pay out-of-pocket for these services. The pandemic has highlighted the urgency for local strategies to support and fund programs that connect OA patients in primary care to community-based physiotherapists for quality nonsurgical care.

In the time it will take to address the backlog of TJR procedures, evidencebased nonsurgical care of hip and knee $\mathrm{OA}$ can reduce pain and improve function and quality of life for those with the disease. Developing collaborative relationships between primary care clinicians in the management of $\mathrm{OA}$ in Canada will vastly improve the management of $O A$ during the pandemic and lay stronger foundations for a culture of team-based primary care in the future.

\section{Allison M. Ezzat PT PhD}

Postdoctoral fellow and physiotherapist, School of Nursing, University of British Columbia, Vancouver, BC

\section{Rita McCracken MD PhD}

Assistant professor and family physician, Department of Family Practice, Faculty of Medicine, University of British Columbia, Vancouver, BC

\section{Sabrina T. Wong RN PhD}

Professor, School of Nursing, University of British Columbia, Vancouver, BC

Cite as: CMAJ 2020 November 16;192: E1471. doi: $10.1503 / \mathrm{cmaj} .76844$

\section{References}

1. Wang J, Vahid S, Eberg M, et al. Clearing the surgical backlog caused by COVID-19 in Ontario: a time series modelling study. CMAJ 2020;192:E1347-56.

2. Hip and knee replacements in Canada: CJRR annual statistics summary, 2018-2019 [report]. Ottawa: Canadian Institute for Health Information; 2020. Available: www.cihi.ca/sites/default/files/ document/cjrr-annual-statistics-hip-knee-2018-2019 -report-en.pdf (accessed 2020 Sept. 1).

3. Kolasinski SL, Neogi T, Hochberg MC, et al. 2019 American College of Rheumatology/Arthritis Foundation Guideline for the management of osteoarthritis of the hand, hip, and knee. Arthritis Rheum 2020;72:220-33.

4. Bannuru RR, Osani MC, Vaysbrot EE, et al. OARSI guidelines for the non-surgical management of knee, hip, and polyarticular osteoarthritis. Osteoarthritis Cartilage 2019;27:1578-89.

5. Skou ST, Roos E, Lauresen M, et al. A randomized controlled trial of total knee replacement. N Engl J Med 2015;373:1597-606.

6. Glazier RH, Badley E, Wright J, et al. Patient and provider factors related to comprehensive arthritis care in a community setting in Ontario, Canada. J Rheumatol 2003;30:1846-50.

7. Davis AM, Kennedy D, Wong R, et al. Cross-cultural adaptation and implementation of Good Life with osteoarthritis in Denmark (GLA:D): Group education and exercise for hip and knee osteoarthritis is feasible in Canada. Osteoarthritis Cartilage 2018; 26:211-9.

8. GLA:D Canada implementation and outcomes: 2019 annual report. Toronto: GLA:D Canada; 2020. Available: gladcanada.ca/wp-content/ uploads/2020/06/2019-GLAD-Canada-Annual-Report _Implementation-and-Outcomes_June-2020-Final .pdf (accessed 2020 Sept. 1).

Competing interests: Allison Ezzat is an instructortrainer with the GLA:D Canada program. 